\section{Age differences in shape recognition through an aperture in a free-viewing situation*}

\author{
JOAN S. GIRGUS \\ The City College of the City University of New York, New York, N.Y. 10031 \\ and \\ JULIAN HOCHBERG \\ Columbia University, New York, N.Y. 10027
}

Young children (ages 4 to 10) show large deficits in correct shape recognition when the shapes are shown piecemeal through an aperture (Girgus \& Hochberg, 1970). This study investigates whether allowing an $S$ to self-program the sequential input results in accurate recognition performance. The stimuli used were a square, a cross, and a block "E." The absence of self-programming in the earlier experiment apparently cannot account fully for the age-related deficits found in that research inasmuch as the results of this experiment still show a significant age effect.

Girgus \& Hochberg (1970) have shown that children, ages 4-10, have difficulty recognizing shapes when those shapes are presented in a piecemeal, sequential fashion through a stationary aperture. This finding is of interest because all of the relevant stimulus information about the shape components and about their relative locations is available in this situation and because the shapes used were easily recognized by all Ss when presented in their entirety under free-viewing conditions. It is reasonable to assume that one or more of the ways in which the aperture-viewing situation differs from the free-viewing one is responsible for the large deficits in shape recognition shown by children under the former conditions. The aperture-viewing technique may, therefore, be a useful tool for investigating how such factors contribute to shape recognition and how they participate in the development of accurate shape processing.

Under the stimulus conditions used by Girgus and Hochberg, the rate and sequence with which the partial views were presented to the $S$ had been chosen by the $E$, whereas, in the free-viewing situation, these are determined by the $S$ himself. The two conditions probably differ, therefore, in rate and sequence of viewing. Moreover, the appearance of each partial view is not preceded by the S's efferent commands to move his eyes in the sequential presentation condition, whereas, in the free-viewing situation, of course, efferent commands to move his eyes necessarily preceded each change in view that the $S$ received. In fact, since the shape was moved

*This research was supported in part by National Institute of Child Health and H um an Development Grants
1R01HD04213-01 and 1R01HDo6002-01. behind a stationary aperture in the sequential presentation condition, the $S$ had to keep his fixation in or near the aperture in order to pick up the pieces of shape information. Thus, there are probably smaller (and perhaps fewer) eye movements made under these conditions than in normal free-scanning situations. The following experiment was conducted in order to ascertain whether the addition of these three factors (self-programmed rate, sequence, and efferent commands) in an aperture-viewing, piecemeal presentation situation would result in accurate shape recognition on the part of young children, or whether age-related deficits would still obtain, showing that additional factors must be assumed to contribute to the deficit in children's performance.

\section{SUBJECTS}

The Ss were 24 elementary school children from a public school in New Jersey. 1 There were $8 \mathrm{Ss}$ in each of three age groups: mean ages 4.6 years, 6.6 years, and 8.5 years, respectively. APPARATUS

The same three test stimuli were used in this experiment as had been used previously: a square ( 1 in. on each side), a cross ( 1 in. on each side), and a block $\mathrm{E}$ ( $5 \mathrm{in}$. high, 2 in. wide, and $1 \mathrm{in}$. on each arm). They were so constructed that the length of one side of the square was equal to one arm of the cross and to the middle arm of the block $\mathrm{E}$. This made the absolute size of the stimuli quite different but equated them in terms of the shortest distance between two corners. The test figure was placed in a holder at the back of a rectangular box, $10 \mathrm{in}$. high $x 9.5$ in. wide $x 21.5$ in. deep. The $S$ looked through a window ( $5 \times 5$ in.) in the front of the box. A partition, 11 in. from the front, divided the box in two. In the center of the partition was a circular hole, $3 / 4$ in. in diam. The same amount of the shape was visible through the hole as was visible through the aperture in the movies used by Girgus \& Hochberg (1970): i.e., approximately one-quarter of the square at any one time. In order to see more than one small piece of the shape, the $\mathbf{S}$ had to move his head around. In this way, the $S$ could make any part of the shape visible through the hole. (This situation is comparable to looking at a scene through a knothole in a fence.) The same matrix of six shapes that was used by Girgus and Hochberg (the three test shapes plus a triangle, a diamond, and a five-pointed star) was used for the recognition procedure of the present study.

\section{PROCEDURE}

A preliminary picture (the head of a boy) was placed in the apparatus, and $\mathrm{S}$ was shown how he could see every part of the figure by moving his head around. The first test stimulus was then inserted, and S was instructed to move his head around so that he saw all of the picture. The three test shapes were each presented twice, in random order. Each presentation lasted $5 \mathrm{sec}$. After each presentation, the $\mathrm{S}$ was shown the confusion matrix and was asked to choose the picture that he had just seen.

Table 1 shows the mean number of correct recognitions per age group and per stimulus. Age was significantly related to the number of correct shape recognitions $[F(2,21)=9.43$, $\mathrm{p}<.011$. However, all age groups recognized, on the average, at least two out of the three test shapes, and the oldest group achieved nearly perfect performance on all three shapes. The differences in correct recognition among the three test stimuli were also significant $[F(2,42)=$ $5.43, \mathrm{p}<.01]$. This latter effect seems to be largely due to differences in the number of correct recognitions between the cross and the block $\mathrm{E}$.

\section{DISCUSSION}

Since children of these ages easily recognize these same test shapes in a free-viewing situation (Girgus \& Hochberg, 1970) yet age was significantly related to the number of correct recognitions in the present

Table 1

Mean Number of Correct Shape Recognitions Per Age Group and Shape*

\begin{tabular}{ccccc}
\hline & & \multicolumn{2}{c}{$\begin{array}{c}\text { Mean Number of Correct } \\
\text { Shape Recognitions }\end{array}$} \\
\cline { 3 - 5 } $\begin{array}{c}\text { Mge in } \\
\text { Years }\end{array}$ & N & & Square Cross & $\begin{array}{c}\text { Block } \\
\text { "E" }\end{array}$ \\
\hline 4.6 & 8 & 1.6 & 1.5 & 0.9 \\
6.6 & 8 & 2.0 & 2.0 & 1.5 \\
8.5 & 8 & 2.0 & 2.0 & 1.9 \\
\hline
\end{tabular}

*Maximum mean number correct $=2$ 


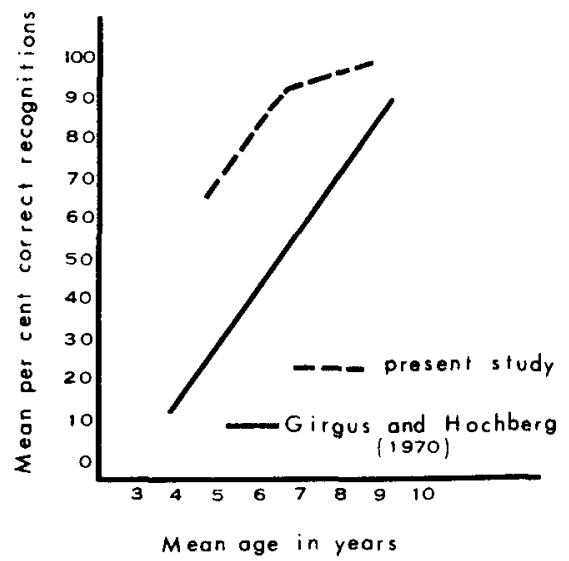

Fig. 1. Percent correct shape recognitions per age group in Girgus \& Hochberg (1970) and in the present study.

study, the viewing conditions used in this study must have failed to provide all of the crucial input characteristics of the normal free-scanning situation. It seems unlikely that the mere fact that sequential presentation was used caused this age-related deficit to occur, since any shape that subtends a visual angle larger than the effective field of view must, of necessity, be processed sequentially. It is probably reasonable to assume that the S's control over the rate and sequence of views, and over the efferent head movement commands that preceded each view, is comparable for the free-viewing situation and the situation used in this study (although we should note that different rates and different movements are probably involved in the two situations). The major way in which all of the aperture-viewing situations used so far differ from free-scanning is that the former fail to provide any information in peripheral vision

It should be noted that the proportions of correct recognitions in this study were somewhat larger than those reported in the previous study. This can be seen visually in Fig. 1 . Unfortunately, no statistical test comparing the two studies is possible, since the $S s$ in the two studies were comparable neither in terms of age nor in terms of the populations from which they were drawn. It is hardly surprising that the figure seems to indicate an advantage for the data from the present study.

In the present situation, the $S$ could determine the order in which he received the pieces of shape information (although the size of each visible piece was determined by the size of the aperture). In addition to determining the order of views in this situation, the $\mathrm{S}$ could decide how much time he wanted to devote to any one particular view. In the 5 sec available to him, he could return to any given view a number of times, spend relatively more or less time looking at it on each fixation, and conceivably could even skip other views altogether. Furthermore, this experiment required $S s$ to issue efferent commands for head movements (and possibly for small, associated eye movements), in order to sample the shape information. These self-initiated movements may contribute to the superior shape recognition performance by furnishing additional information as to the position of each view, relative to the other views. However, it should be noted that, with all of the advantages that this situation provides as contrasted with the aperture-viewing situation used by Girgus \& Hochberg (1970), the age-related differences found in the latter are also obtained here.

\section{REFERENCE}

GIRGUS, J. S., \& HOCHBERG, J. E. Age differences in sequential form recognition. Psychonomic Science, 1970, 21, 211-212.

\section{NOTE}

1. The authors would like to thank the principal, teachers, and students of the experimental classes at the Educational Resource Center of the Institute of Child Study of Newark State College, Newark, N.J. 